\title{
In vivo comparison of pulsed versus continuous wave Nd:YAG laser photoablation
}

\author{
A. SIBILLE, C. D'ALINCOURT ${ }^{* *}$, T. PONCHON, F. BERGER* and R. LAMBERT \\ Department of Digestive Diseases, Edouard Herriot Hospital, 69437 Lyon, France \\ ${ }^{*}$ Department of Pathology, Edouard Herriot Hospital, 69437 Lyon, France \\ ** Technomed International, 69500 Bron, France
}

Two modes of Nd: YAG laser are available for medical and surgical applications. With the continuous wave (CW) mode the power selected is distributed continuously during emission. With the pulsed wave (PW) mode, high power pulses of short duration are delivered at a constant frequency. The duration of the pulses is depending on the selected mean power.

From previous published studies it may be assumed that the tissue necrosis and crater induced with the CW mode are deeper than with the PW mode $(1,2)$. The theoretical advantage of the PW mode is therefore better control of the necrosis extension.

The aim of this study was to compare, for the same energy levels, the necrosis induced by the two modes of emission of the Nd:YAG laser in the rat liver in vivo.

\section{MATERIAL AND METHODS}

\section{1-Laser}

The laser source used was a medical Nd:YAG laser, the Multilase 2500 (Technomed International, Bron, France), which may operate either in the continuous wave (CW) or in the pulsed wave (PW) mode, at $1064 \mathrm{~nm}$. With the $\mathrm{CW}$ mode the selected power was continously delivered during the shot. The maximum power was $100 \mathrm{~W}$. With the PW mode high power pulses of $300 \mathrm{~W}$ were delivered with a repetition frequency of $10 \mathrm{~Hz}$. The mean power was tunable by modifying the pulses duration between 1 and $30 \mathrm{msec}$.

\section{2-Animals and design of experiments}

Wistar rats weighing 500-600 g (Ifa-Credo, L'Abresle, France) were anaesthetized by an intraperitoneal injection of sodium pentobarbital $(0.1 \mathrm{ml} / \mathrm{kg})$. After laparotomy, single laser shots were performed separately on the liver surface, using a $400 \mu \mathrm{m}$ quartz fiber. $4-5$ shots was performed for each parameter combination with each laser modes.

In study 1 , the quartz fiber was handled close to the liver surface ('contact' method). The parameters combinations (power and exposure time) tested were as follows : 10W-1sec, $10 \mathrm{~W}$ $2 \mathrm{sec}, 20 \mathrm{~W}-1 \mathrm{sec}, 20 \mathrm{~W}-2 \mathrm{sec}$. Fluences were $7958,15915,15915$ and $31831 \mathrm{~J} / \mathrm{cm}^{2}$, respectively.

In study 2 , the quartz fiber was fixed at a distance from the liver and the laser beam was refocused through a handpiece, to obtain a spot of $3 \mathrm{~mm}$ in diameter at the liver surface ("non contact' method). The parameters combinations (power and exposure time) tested were as follows : $20 \mathrm{~W}-1 \mathrm{sec}, 20 \mathrm{~W}-2 \mathrm{sec}, 40 \mathrm{~W}-1 \mathrm{sec}, 40 \mathrm{~W}-2 \mathrm{sec}$. Fluences were 283, 566, 566 and 1132 $\mathrm{J} / \mathrm{cm}^{2}$, respectively. 


\section{3-analysis of results}

After excision, the liver was fixed in formalin. Serial slices along the laser beam axis were stained with hematoxylin, eosin and saffron. Maximal depth and diameter of the three areas that may be oserved in a laser-induced tissue lesion (crater, necrosis and congestion) (3) were measured for each lesion, through microscopic examination with a graduated eye-piece. Volumes were then calculated.

Comparisons of mean results (CW versus $\mathrm{PW}$ ) for each parameter combination was done with the Student t-test.

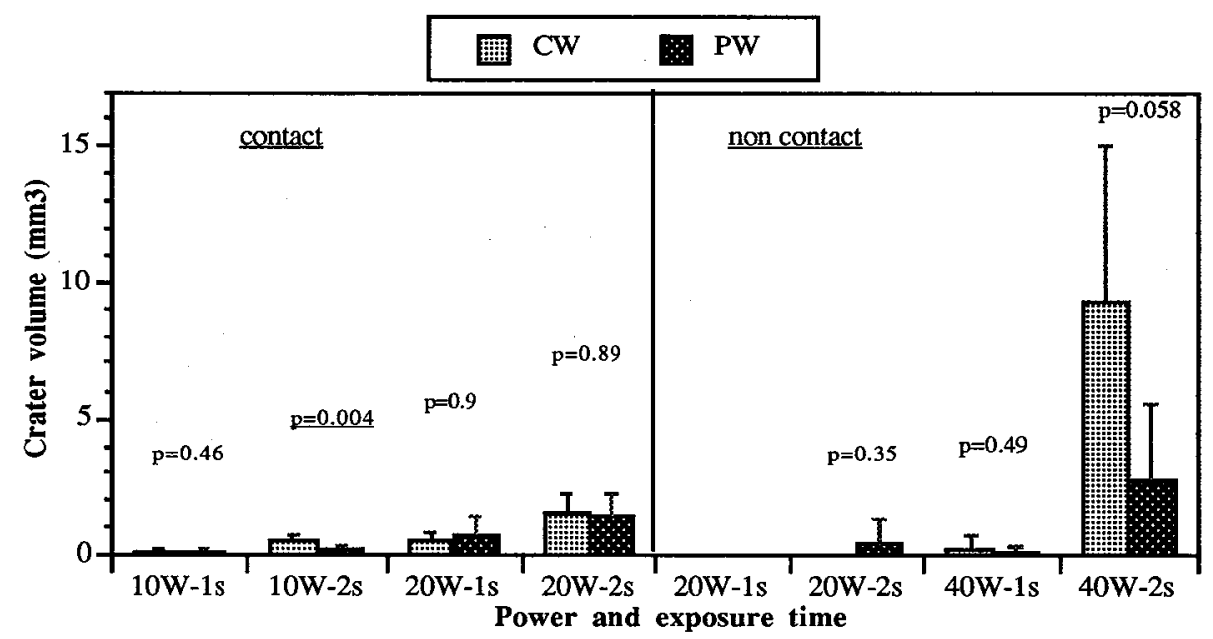

Fig 1: Crater volumes of $\mathrm{CW}$ vs $\mathrm{PW}$ lesions according to each parameter

\section{RESULTS}

Figures 1 to 3 show the volumes of lesion -crater (Fig 1), necrotic area (Fig 2), total volume (Fig 3)- induced by the CW versus the PW mode for each parameter combination in the two studies.

Study 1 (contact) :

When considering either volume or depth of the three areas (crater, necrosis, congestion) of induced lesions, no significant difference was observed between the two modes of Nd:YAG laser, excepted at $10 \mathrm{~W}-2 \mathrm{sec}\left(15915 \mathrm{~J} / \mathrm{cm}^{2}\right)$ where the crater volume and depth induced by the CW mode were significantly larger than these obtained with the PW mode $(\mathrm{p}=0.004)$.

Study 2 (non contact) :

The necrosis volume, total volume and total depth of the lesion induced by the PW mode were 
significantly larger than those obtained with the $\mathrm{CW}$ mode at the fluence of $566 \mathrm{~J} / \mathrm{cm}^{2}$, but this difference was not observed at the other fluences. There was no difference in the crater volume or depth.

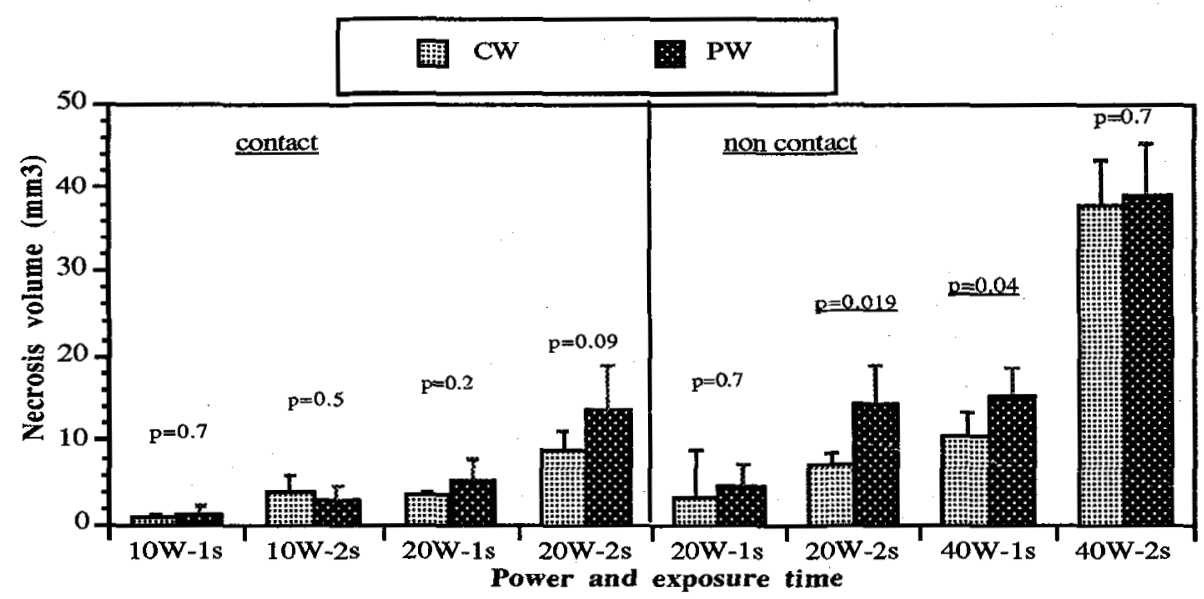

Fig 2: Necrosis volumes of $\mathrm{CW}$ vs $\mathrm{PW}$ lesions according to each parameter

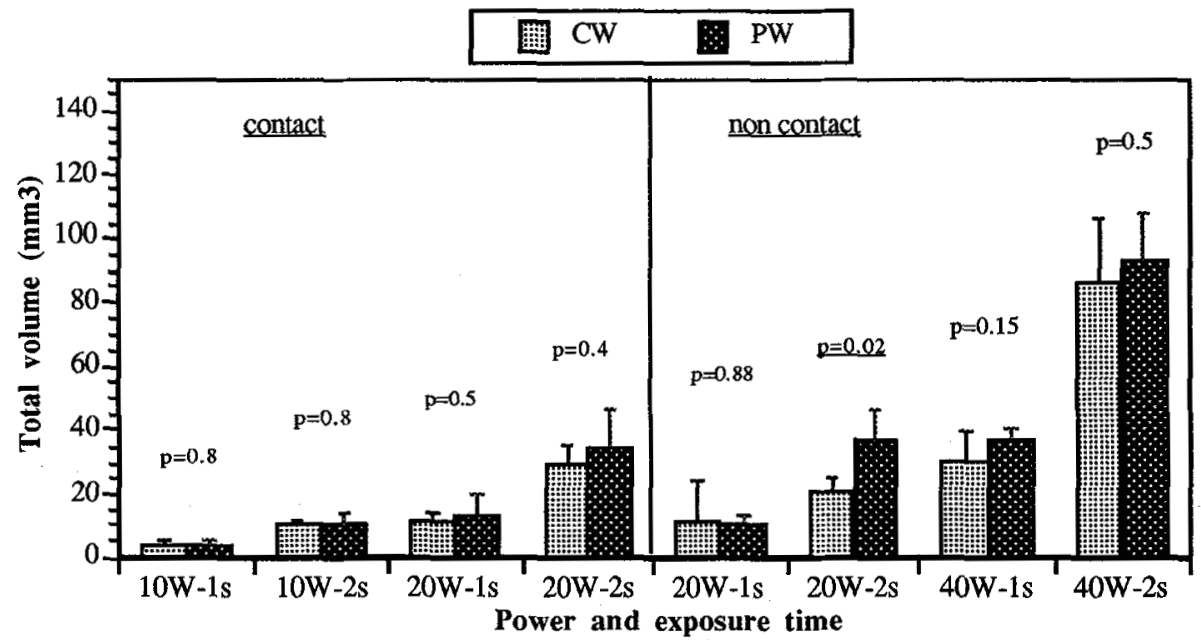

Fig 3: Total volumes of CW vs $\mathrm{PW}$ lesions according to each parameter 


\section{DISCUSSION}

This study did not show great difference in the tissue necrosis induced by the two modes of emission of the Nd:YAG laser, in the rat liver in vivo. The only differences obtained at a precise fluence were surprisingly the formation of a larger crater with the CW mode in study 1 and the induction of a deeper tissue necrosis by the PW mode in study 2 . This trend is in opposition with the results of Mordon (1) and Rebeiz (2). The former study showed that with the pulsed Nd:YAG laser the depth of induced necrosis in the perfused tissue was smaller than with the CW mode. This study was performed with the same model as our's, but with a different kind of pulsed laser (peak power : $500 \mathrm{~W}$, variability of pulse and pulse-to-pulse durations). In addition, Mordon wanted to avoid vaporization, and rejected $10 \%$ of its data because the vaporization threshold was reached. In the present study, the two modes were compared at several fluences and all data were analyzed without regard to vaporization. Indeed, there is a relative variability of results in living tissues studies even in strictly comparable experimental conditions (3) and sometimes vaporization occurs during a shot and not during another performed at the same fluence. Another feature that may explain these controversial findings is the higher peak power of the pulsed laser in the Mordon's study.

Rebeiz (2) used the same pulsed Nd:YAG laser as we. He found that the CW mode created deeper lesions and more extensive damage around the crater than with the PW mode. However, the power used with the CW mode was higher than with the PW mode, a fact that impeded interpretation of the results. In addition, these experiments were done in the tracheal wall, during an endoscopic procedure : with such a device, it seems difficult to keep the laser beam strictly perpendicular to the target and obtain accurate and reproductible measurements of the lesion depth.

In conclusion, the present study performed in a homogenous perfused tissue did not show a great difference in depth and volume of lesion induced by both mode of the Nd:YAG laser, excepted at the fluence of $566 \mathrm{~J} / \mathrm{cm}^{2}$ where the PW mode induced necrosis was deeper.

\section{REFERENCES}

1. Mordon SR, Cornil AH, Brunetaud JM, Gosselin B, Moschetto Y. Nd-YAG laser thermal effect : comparative study of coagulation in rat liver in vivo by continuous wave and high power pulsed lasers. Lasers Med Sci 1987;2:285-294.

2. Rebeiz EE, Aretz T, Shapshay SM, Pankratov MM. Application of pulsed and continuous wave 1.32 and $1.06 \mathrm{~mm}$ wavelengths of the Nd:YAG laser in the canine tracheobronchial tree : a comparative study. Lasers Surg Med 1990;10:501-509.

3. Brackrett KA, Sankar MY, Joffe SN. Effects of Nd:YAG laser photoablation on intra-abdominal tissues : a histological study of tissue damage versus power density applied. Lasers Surg Med 1986;6:123-130 